\title{
Vector quality measure for vector beams
}

Sroor, Hend, Ndagano, Bienvenu, McLaren, Melanie, Rosales-Guzman, Carmelo, Forbes, Andrew

Hend Sroor, Bienvenu Ndagano, Melanie McLaren, Carmelo RosalesGuzman, Andrew Forbes, "Vector quality measure for vector beams," Proc. SPIE 9950, Laser Beam Shaping XVII, 99500F (27 September 2016); doi: 10.1117/12.2237695

SPIE Event: SPIE Optical Engineering + Applications, 2016, San Diego, California, United States 


\title{
Quality factor of vector beams
}

\author{
Hend Sroor $^{a}$, Bienvenu Ndagano ${ }^{a}$, Melanie McLaren ${ }^{a}$, \\ Carmelo Rosales-Guzman ${ }^{a}$ and Andrew Forbes ${ }^{a}$ \\ a School of Physics, University of the Witwatersrand, Johannessburg, South Africa.
}

\begin{abstract}
Vector beams are spatial modes of light with spatially variant polarization states in the transverse profile. Over the years, vector beams have found their way into plenty of applications ranging from material processing and lithography to electron acceleration and particle trapping. Though qualitative measurements are routinely used to analyse vector beams, there is currently no quantitative measure for vector beam purity. Here, we introduce a new measure, the vector quality factor (VQF), that maps the purity of vector beams to a scale ranging from 0 to 1. We demonstrate a simple optical setup to generate and detect vector beams using a birefringent phase plate known as a q-plate. Tomographic measurements are performed by decomposing the vector beam into its circular basis states, and measuring the expectation values of the Pauli matrices as intensity measurements which, are used to evaluate the VQF of vector beams.
\end{abstract}

Keywords: vector beams, modal decomposition, quality factor, entanglement

\section{INTRODUCTION}

In the past two decades, there has been a growing interest in utilizing spatial modes of light in areas ranging from optical communication ${ }^{1-4}$ to optical manipulation of microscopic particles. ${ }^{5-8}$ In general, spatial modes of light can be grouped in two categories: scalar modes and vector modes. The former is more familiar to many in the field; these are spatial modes of light with uniform polarization, i.e., the spatial and polarization degree of freedom are independent. Laser modes such as Laguerre-Gaussian or Hermite-Gaussian modes are two examples of scalar modes. Though their optical field can often be qualitatively characterized by visual inspection of the intensity profile, a more accurate, quantitative description, is provided by the $\mathrm{M}^{2}$ propagation ratio introduced by A. Siegman. Treating the optical field (independently of the polarization) as a probability wave, the $\mathrm{M}^{2}$ ratio can be derived from second moments of the intensity. ${ }^{9}$ In recent work by Schulze et al., it was shown that $\mathrm{M}^{2}$ measurements could be performed digitally with no moving component, as is typically the case. ${ }^{10}$ For vector beams however, such as measure is inadequate.

Vector beams are spatial modes of light with non-uniform polarization distribution; ${ }^{11}$ that is, the polarization varies depending on the position across the beam profile. They can typically be represented as incoherent superposition of optical fields, added in a non-separable fashion, hence why one cannot assign a single $\mathrm{M}^{2}$ value to the vector mode in general. Though vector modes can routinely be produced with beam shaping techniques involving dynamic and geometric phase manipulations, inside and outside laser cavities. ${ }^{12-14}$ It has become common standard to measure the state of the vector beam by observing the behaviour of the intensity pattern through a rotating polarizer; as the polarizer rotates, the intensity pattern observed varies. Based on the variation of the observed pattern, one then infers whether the beam under investigation is a vector or a scalar beam. What if the beam were to not be fully scalar nor fully vector? A simple visual observation would be, inadequate and inaccurate. Here, we demonstrate techniques to generate and measure, quantitatively, the state of 'vectorness' of an arbitrary vector beam. This approach takes advantage of the non-separability that is inherent to vector beams, a property traditionally associated with the entanglement of quantum states, for the measurement tools are very well established in quantum mechanics. Hence, just as Siegman borrowed statistical tools to derive the beam propagation ratio, we borrow quantum tools to measure the degree of non-separability or vectorness of a general optical field. We assign a single value between 0 and 1 to the state of vectorness: 0 for purely scalar and 1 for purely vector.

Further author information: (Send correspondence to A. Forbes) E-mail: andrew.forbes@wits.ac.za 


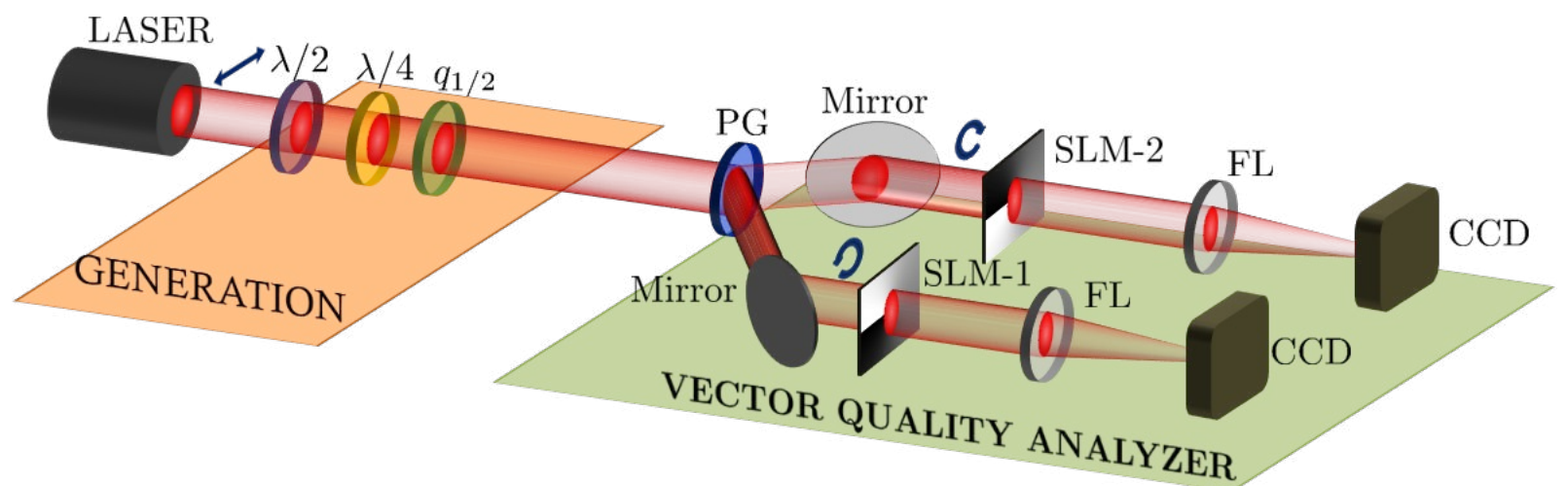

Figure 1: Experimental generation and analysis of vector modes. Using a half-wave plate $(\lambda / 2)$ to adjust the polarization of the fundamental Gaussian mode, we generated a radially (or azimuthally) polarized vector mode with a q-plate of topological charge $q=1 / 2$, and directed it onto a vector quality analyzer system, where the quality factor is measured by performing a series of polarization projections using a polarization grating (PG), and OAM projections encoded on the spatial light modulator (SLM), measuring the outputs in the Fourier plane of two Fourier lenses with two CCDs.

\section{THEORY}

Considering an arbitrary transverse mode -mapped onto the surface of the HOP sphere by $\theta$ - represented by

$$
V(r, \theta)=\cos (\theta)\left|V_{L}(r)\right\rangle\left|e_{L}\right\rangle+\sin (\theta)\left|V_{R}(r)\right\rangle\left|e_{R}\right\rangle
$$

where $\left|e_{L}\right\rangle$ and $\left|e_{L}\right\rangle$ represent the left and right polarization state associated to spatial modes $\left|V_{L}(r)\right\rangle$ and $\left|V_{R}(r)\right\rangle$ respectively. We posed important questions; what should a suitable measure be to determine if the beam is vector or not, and how can this be measured?

We mentioned above that the non-separability of vector beams is analogous to the entanglement of quantum states. In quantum mechanics, there are multiple measures of entanglement, derived for bipartite systems similar to that described as in Eq. 1. Here, we choose the concurrence as our measure of entanglement (nonseparability). ${ }^{15,16}$ We defined our vector quality factor in terms of the concurrence as follows:

$$
\mathrm{VQF}=\operatorname{Re}(C)=\operatorname{Re}(\sqrt{1-h}),
$$

where the length of the Bloch vector is defined as

$$
h=\left(\sum_{i=1}^{3}\left\langle\sigma_{i}^{2}\right\rangle\right)^{1 / 2} .
$$

Here $\left\langle\sigma_{i}\right\rangle$ is the Pauli operator and optically corresponds to the degree of polarization in the average polarization state. $^{17}$

For example, consider a radially polarized vector beam of the form

$$
V=\frac{1}{\sqrt{2}}\left(\exp (i \ell \phi) e_{R}+\exp (-i \ell \phi) e_{L}\right),
$$

where $\ell$ is the azimuthal index of the beam carrying $\ell \hbar$ orbital angular momentum (OAM) per photon. To calculate $h, 12$ intensity measurements, six for both circular polarization states, are required. The Pauli operators can be defined as

$$
\begin{aligned}
& \left\langle\sigma_{1}\right\rangle=\left(I_{1,3}+I_{2,3}\right)-\left(I_{1,5}+I_{2,5}\right), \\
& \left\langle\sigma_{2}\right\rangle=\left(I_{1,4}+I_{2,4}\right)-\left(I_{1,6}+I_{2,6}\right), \\
& \left\langle\sigma_{3}\right\rangle=\left(I_{1,1}+I_{2,1}\right)-\left(I_{1,2}+I_{2,2}\right) .
\end{aligned}
$$


Here, $i$ is chosen as the circularly polarization basis $\{L, R\}$, while $j$ represents two pure OAM states, $l,-l$, and four OAM superposition state, $u=\exp (i \ell \phi)+\exp (i \theta) \exp (i \ell \phi)$, with an intermodal phase of $\theta=0, \pi / 4, \pi / 2,3 \pi / 2$. These six OAM measurements map the HOP sphere. This technique is an adaptation of a full-state tomography that is more commonly used in quantum entanglement, where an over-complete set of measurements is used to describe the full density matrix.

\section{EXPERIMENT}

Experimentally, this has been implemented by employing a combination of geometric and dynamic phase control. ${ }^{18}$ The vector beams are generated using a q-plate as shown in Figure (1). The q-plate follows the selection rules(written in bra-ket notation): $|l, L\rangle \rightarrow|l+2 q, R\rangle$ and $|l, R\rangle \rightarrow|l-2 q, L\rangle$, where q is the azimuthal charge introduced by the q-plate $(q=1 / 2)$. Regardless of the generation technique, the vector mode can be analysed in a vector quality analyzer system. The vector quality analyzer system make use of a modal decomposition technique, ${ }^{19}$ where an inner product of the incident field with a match filter is used to determine the weighting coefficients of the mode. That is, the modal decomposition of the input field $u$ into the azimuthal modes $\exp (i \ell \phi)$ such that $u=\sum_{\ell} a_{\ell} \exp (i \ell \phi)$, the modulus of the modal weighting coefficients $a_{\ell}$ can be determined by the inner product of the field with an azimuthal match filter: $|\langle u \mid \exp (i \ell \phi)\rangle|=\left|a_{\ell}\right|$. Optically, the inner product is performed by directing the incident beam onto a match filter (typically encoded onto a spatial light modulator) and viewing the Fourier transform, with the use of a lens (FL), on a CCD camera.

The vector quality factor does not depend on the type of vector beam and can be determined by performing measurements on both the polarization and OAM degrees of freedom. The polarization component was measured using a polarization grating $(\mathrm{PG})$, which diffracts light into two beams in the +1 and -1 orders such that the two output beams have opposite circular polarizations. Each polarization arm was directed onto a spatial light modulator (SLM) encoded with a digital phase pattern that acts as the azimuthal match filter for the decomposition. In order to satisfy Eqs. (5 - 7), the on-axis intensity for six different OAM states were measured in each polarization arm. Figure 2(a) and (b) show the twelve normalized intensity measurements for a vector and scalar vortex beam, respectively. In this case, the circular polarization basis was chosen and six different OAM projections were made for both the left- and right-circular polarization states. Phase-only holograms were encoded onto the SLM to detect the azimuthal component of the vortex modes.

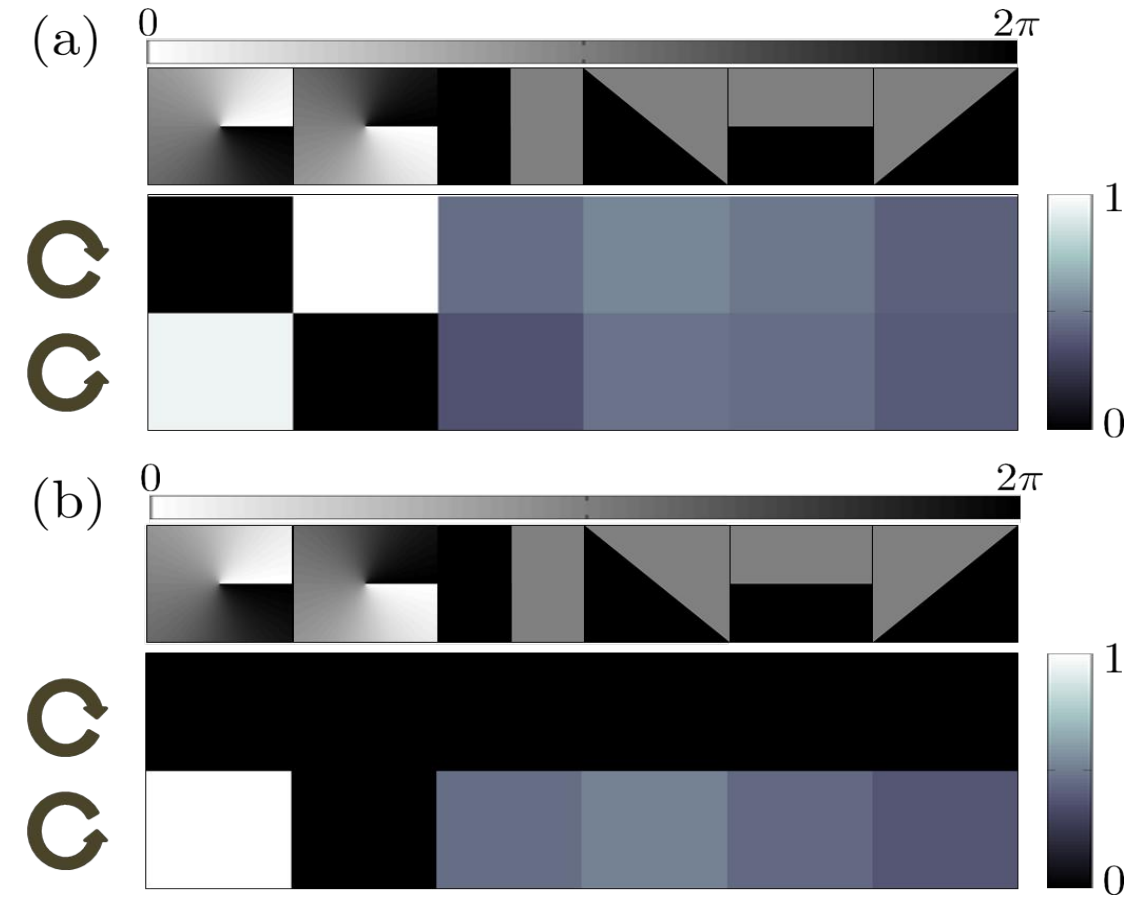

Figure 2: Normalized on-axis intensity measurements of OAM projections in each circular polarization state for (a) a vector vortex mode and (b) a scalar vortex mode. 
From the intensity measurements, the VQF for a radially polarized vector vortex beam was calculated, using Eq. 2 , to be $0.98 \pm 0.01$. By simply rotating the quarter-wave plate $(\lambda / 4)$ before the first q-plate in the generation section, the vortex beam created can be varied from a vector to a scalar beam. As such, the VQF was measured for different orientations of the quarter-wave plate and the results, graphically illustrated in Fig. 3, show that this measurement of the vector quality maps purely scalar through to purely vector beams with the range 0 through 1.

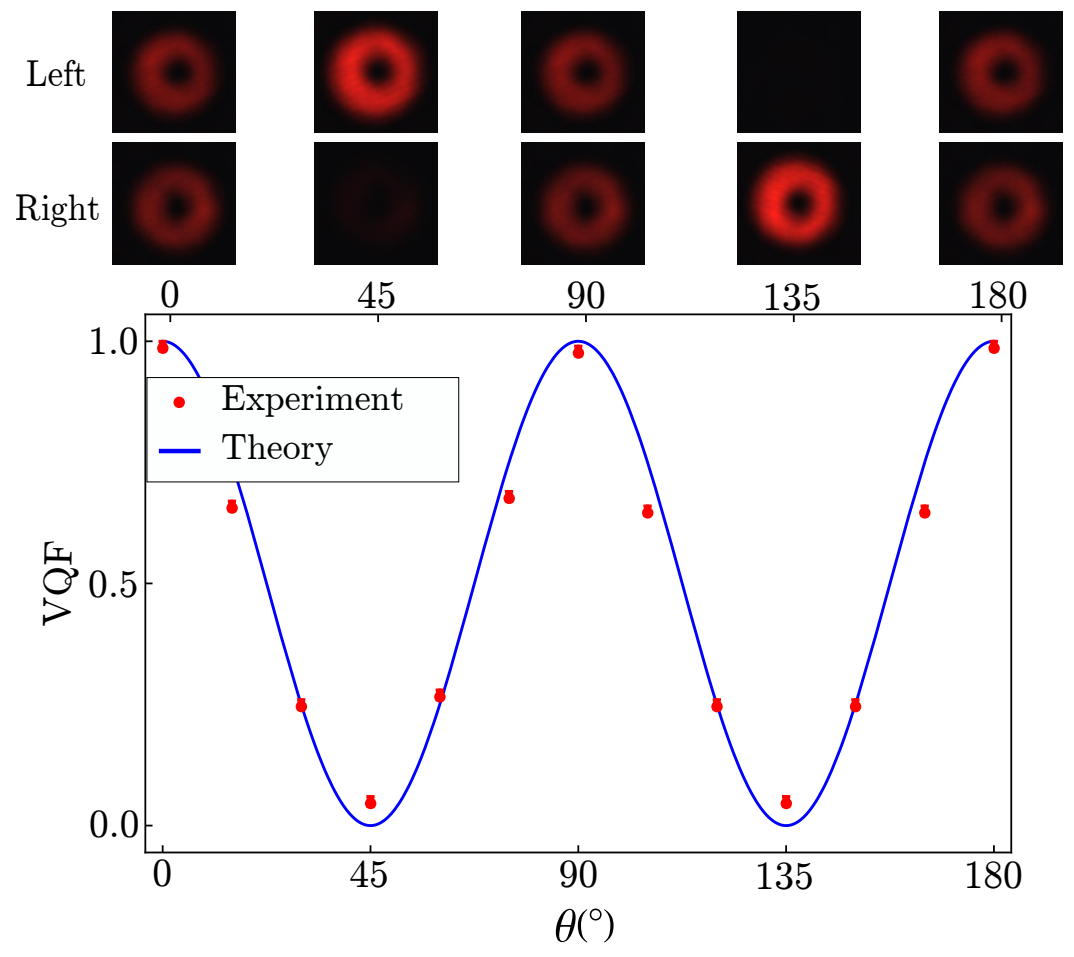

Figure 3: Evolution of the vector quality factor (VQF) with respect to the nature of the input state. The VQF was calculated from the 12 decomposition measurements (red dots) and plotted against the theoretical prediction (blue solid line) for each orientation of the quarter-wave plate.

We point out here that while our implementation has been based on vector vortex beams, this is for convenience only. The concept and definition of a vector beam quality factor is independent of the type of vector beam under study, and only the optical elements in the measurement setup would require change (we anticipate that many users will require a measure for a known form of vector beam). Moreover, this approach produces a single number that immediately indicates how vector the mode is, i.e., a quantitative measure. In contrast, the degree of polarization produces a number for every point on the field, and thus while this is quantitative for a particular point, it does not determine the vector quality of the mode as a whole. We would also like to highlight that the approach is independent of the form of the vector beam.

In conclusion, we have introduced a new laser beam characterization tool for arbitrary vector beams. We have demonstrated the concept on cylindrical vector vortex beams with varying degree of vector quality, from purely scalar (OAM modes) to purely vector (radial and azimuthally polarized light), and shown excellent agreement between the predicted and measured vector quality factors. Our vector quality factor offers a quantitative measure of the quality of a vector mode, which we believe will be a useful tool in the analysis of such beams in both laboratory and industrial applications.

\section{REFERENCES}

[1] Brüning, R., Ndagano, B., McLaren, M., Schröter, S., Kobelke, J., Duparré, M., and Forbes, A., "Data transmission with twisted light through a free-space to fiber optical communication link," Journ. of Opt. 18(3) (2016). 
[2] Ndagano, B., Brüning, R., McLaren, M., Duparré, M., and Forbes, A., "Fiber propagation of vector modes," Opt. Exp. 23(13), 17330 (2015).

[3] Trichili, A., Rosales-Guzmán, C., Dudley, A., Ndagano, B., Ben Salem, A., Zghal, M., and Forbes, A., "Optical communication beyond orbital angular momentum," Sci. Rep. 6(1), 27674 (2016).

[4] Cox, M. A., Rosales-Guzmán, C., Lavery, M. P. J., Versfeld, D. J., and Forbes, A., "On the resilience of scalar and vector vortex modes in turbulence," Opt. Exp. 24(16), 18105-18113 (2016).

[5] Grier, D. G., "A revolution in optical manipulation.," Nat. 424(6950), 810-816 (2003).

[6] Kozawa, Y. and Sato, S., "Optical trapping of micrometer-sized dielectric particles by cylindrical vector beams," Opt. Exp. 18(10), 10828-10833 (2010).

[7] Padgett, M. and Bowman, R., "Tweezers with a twist," Nat. Phot. 5(6), 343-348 (2011).

[8] Donato, M., Vasi, S., Sayed, R., Jones, P., Bonaccorso, F., Ferrari, A., Gucciardi, P., and Maragò, O., "Optical trapping of nanotubes with cylindrical vector beams," Opt. Lett. 37(16), 3381-3383 (2012).

[9] Siegman, A. E., "Defining, measuring, and optimizing laser beam quality," in [Laser Applications in Science\&3 Engineering], 2-12, SPIE (1993).

[10] Schulze, C., Flamm, D., Duparré, M., and Forbes, A., "Beam-quality measurements using a spatial light modulator," Opt. Lett. 37(22), 4687-4689 (2012).

[11] Zhan, Q., "Cylindrical vector beams: from mathematical concepts to applications," Adv. Opt. Phot. 1(1), $1-57(2009)$.

[12] Naidoo, D., Roux, F. S., Dudley, A., Litvin, I., Piccirillo, B., Marrucci, L., and Forbes, A., "Controlled generation of higher-order poincaré sphere beams from a laser," Nat. Phot. 10(5), 327-332 (2016).

[13] Bell, T., Äit-Ameur, K., Forbes, A., and Ngcobo, S., "Intracavity generation of low-loss radial-order laguerregaussian modes using digital holograms," in [SPIE LASE], 97271K-97271K, SPIE (2016).

[14] Forbes, A., Dudley, A., and McLaren, M., "Creation and detection of optical modes with spatial light modulators," Adv. Opt. Phot. 8(2), 200-227 (2016).

[15] Hill, S. and Wootters, W. K., "Entanglement of a Pair of Quantum Bits," Phys. Rev. Lett. 78(26), 5022-5025 (1997).

[16] Wootters, W. K., "Entanglement of Formation of an Arbitrary State of Two Qubits," Phys. Rev. Lett. 80(10), 2245-2248 (1998).

[17] McLaren, M., Konrad, T., and Forbes, A., "Measuring the nonseparability of vector vortex beams," Phys. Rev. A 92(2), 023833 (2015).

[18] Ndagano, B., Sroor, H., McLaren, M., Rosales-Guzmán, C., and Forbes, A., "Beam quality measure for vector beams," Opt. Lett. 41(15), 3407-3410 (2016).

[19] Flamm, D., Hou, K.-C., Gelszinnis, P., Schulze, C., Schröter, S., and Duparré, M., "Modal characterization of fiber-to-fiber coupling processes," Opt. Lett. 38(12), 2128-2130 (2013). 